\title{
Reversible Hardware for Acoustic Communications
}

\author{
Harun Siljak, Member, IEEE, Julien de Rosny, Member, IEEE and Mathias Fink
}

\begin{abstract}
Reversible computation has been recognised as a potential solution to the technological bottleneck in the future of computing machinery. Rolf Landauer determined the lower limit for power dissipation in computation and noted that dissipation happens when information is lost, i.e., when a bit is erased. This meant that reversible computation, conserving information conserves energy as well, and as such can operate on arbitrarily small power. There were only a few applications and use cases of reversible computing hardware. Here we present a novel reversible computation architecture for time reversal of waves, with an application to sound wave communications. This energy efficient design is also a natural one, and it allows the use of the same hardware for transmission and reception at the time reversal mirror.
\end{abstract}

Index Terms-reversible computation, circuit design, wave time reversal, wireless communications

\section{INTRODUCTION}

$\mathbf{T}$ HE majority of computation we perform is irreversible: addition of two numbers, or logical AND of two bits both destroy the information about the inputs. In a computation paradigm which prioritises saving memory resources, losing information is a consequence of using a register for something else as soon as its current content is used for the last time. The arithmetic units are often designed so that the result replaces one of the input operands, and this is considered an important save in resources. Somewhat paradoxically, the call for reversibility and preservation of information through the computation process also comes from the resource optimization perspective.

Thermodynamics of computation explains the mechanisms of energy use and dissipation in computing systems. Landauer [1] established an important lower limit for computation energy dissipation: the erasure of one bit takes a fraction of a joule, energy proportional to the working temperature of the system (with the proportionality coefficient equal to the product of the Boltzmann constant and natural logarithm of two). This lower limit follows from the equivalence of thermodynamical and informational entropy and, at the time, it was significantly lower than the limits imposed by technological (semiconductor) constraints. With the advancement of semiconductor industry, the limits of devices became closer to Landauer's limit. Landauer's limit bounds bit erasure:

This publication has emanated from research supported in part by a research grant from Science Foundation Ireland (SFI) and is co-funded under the European Regional Development Fund under Grant Number 13/RC/2077. The project has received funding from the European Unions Horizon 2020 research and innovation programme under the Marie Skodowska-Curie grant agreement No 713567 and was partially supported by the COST Action IC1405.

Harun Siljak is with CONNECT Centre, Trinity College Dublin, Ireland, e-mail: siljakh@tcd.ie.

Julien de Rosny and Mathias Fink are with Institut Langevin, ESPCI ParisTech, CNRS UMR 7587, 75231 Paris Cedex 05, France, e-mails: \{julien.derosny,mathias.fink\} @espci.fr. operations that do not erase information (bits) do not have a lower bound in thermodynamical-informational sense. This fact qualifies information-conserving computation as a potential solution for the future of general computing in the postMoore law era.

The concept of reversible computation, reversible logic gates and circuit design have been a topic of research since Bennett's pioneering work [2] on applying Landauer's ideas to hardware. However, there have been almost no applications of reversible circuits to real world problems, no interfaces with the nature and other technology. In this paper, we present a case for employing reversible computation in wave time reversal, using acoustic underwater communication as a working example.

The case of acoustic communications based on wave time reversal is a good ground for reversible computation. Everything is reversible: the communication scheme reversing the carrier wave toward the original source and the environment obeying reversible Euler equation. Here we show how the computation performing all of it can be reversible as well. Of course, wave time reversal is not limited to acoustic communication, as it is the basis of a beamforming approach for RF communications. Hence, our contribution is relevant to multiple wireless communications paradigms. Our motivation for presenting the reversible hardware solution for wave time reversal as a contribution to communications stems from here: a solution for time-reversal massive MIMO would be an adaptation of the one presented here, and same holds for optical communications based on time reversal. In this manner, digital signal processing in communications would be ready for the post-Moore age of reversible and/or quantum computing. Time-reversal based communications implemented with this circuitry then have both the potential of immense energy efficiency and a chance to become the natural solution for the physical layer of quantum networks of the future.

We begin by revisiting the mechanism of wave time reversal, followed by a presentation of reversible computation and motivation of its use. We discuss the possible design options employing reversible hardware for time reversal and show design results. We conclude with a discussion of the proposed solution, future research and the wider effect of reversible hardware introduction in wireless communications.

\section{REVERSIBILITY OF WAVES AND COMPUTATION}

It is not a coincidence that we chose wave time reversal for the demonstration of an efficient reversible hardware application. As this section will show, the wave time reversal and reversible computation both rely on keeping the information about backtracking known, to run backwards. They share the same philosophy and a common foe. 


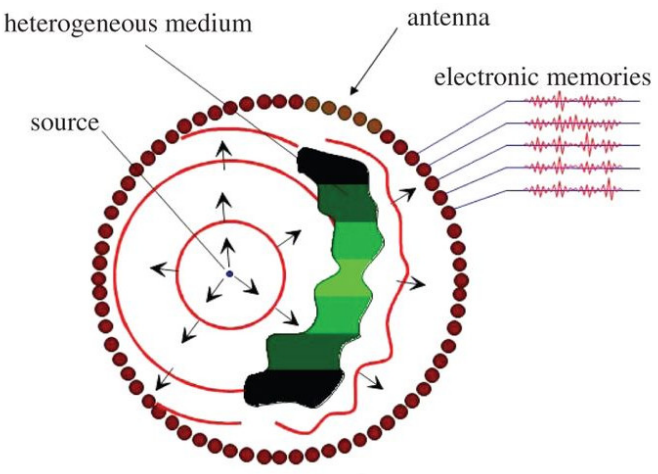

Fig. 1. The wavefront distorted by heterogeneities comes from a point source and is recorded on the cavity elements. In the next step the recorded signals are time-reversed and re-emitted by the elements. The time-reversed field back-propagates and refocuses exactly on the initial source.[3]

\section{A. Wave Time Reversal}

Both, reversing waves and reversing computation are plagued with the scale destroying reversibility. While at the microscale the elementary components of the system are obeying reversible laws (be it simple computational operations, be it equations of motion), their ensembles lose the reversibility at the macroscale. Loschmidt's thought experiment with a deamon capable of reversing all velocities of particles in a gas and hence reversing the behaviour of the ensemble asks for too much information and ability on the deamon's side, but it is a worthy goal to pursue: how can we reverse a propagating wave so it ends up converging at its original source?

The solution based on time reversal mirrors (TRMs) [4] performs this regardless of the complexity of the medium as if time were going backwards, and has been implemented with acoustic, electromagnetic and water waves. It requires the use of emitter-receptor antennas positioned on an arbitrary enclosing surface. The wave is recorded, digitized, stored, time-reversed and rebroadcasted by the same antenna array. If the array intercepts the entire forward wave with a good spatial sampling, it generates a perfect backward-propagating copy.

The principle of wave time reversal builds upon the exact nature of the wave equation, and its solution being a continuous function of three spatial and one temporal dimension, i.e. described over a hypervolume with four variables, bounded by a hypersurface with three variables. This boundary can either be observed as composed of three spatial dimensions or of two spatial and one temporal dimension . Depending on the choice of the boundary description, we have two different approaches to time reversal, dubbed "à la Huygens" (named after Huygens integral theorem) and "à la Loschmidt" (named after Loschmidt's deamon). [3]

1) The time-reversal mirror approach 'à la Huygens': In this approach, represented in the Fig. 1, a transient wavefield originating from the initial source is radiated throughout a heterogeneous medium closed in a cavity bounded by a twodimensional surface. This surface is populated with sensing and recording devices keeping the information about the wavefield and its normal derivative. This process continues until the incoming field vanishes along the boundary. This recording suffices for the recovery of the wavefield, as we will soon see.

Out of the two solutions of the wave equation (wave operator obeys time-reversal symmetry), the causal one is radiated from the source, and we aim to radiate the anti-causal one from the boundary. The collected samples are hence timereversed and rebroadcasted by the same antenna array that has collected them.

This new wave satisfies a homogeneous wave equation with the time-reversed boundary conditions without the original source. Hence it is not enough to time-reverse the wavefield on the boundary, as the original source needs to be reversed into a sink. While returning to the original source, the re-emitted wave does appear to converge, but as it cannot stop on its own, after the collapse it continues to propagate in divergent manner. To compensate this diverging field, we either use an active source at the focusing point canceling the field, or a passive sink as a perfect absorber. [5]

So far, we assumed the idealised case where the entire surface of the boundary is covered with transceivers, which requires a large number of hardware components. This requirement can be dropped, and the execution of the reversal can be simplified. One way is locating the TRM in the far field of the source and of the medium heterogeneities. This halves the quantity of data to be stored, as the normal derivative remains proportional to the field and does not have to be recorded at all. In addition to this, it was experimentally shown that TRM consisting of a small number of elements (time-reversal channels) functions on a limited angular area as well, when it uses complex environments to appear wider than it is. The resulting refocusing quality does not depend on the TRM aperture. In that regard, observe the following experiment setup.[6] A point-like transducer is separated from a TRM by a large distance (much larger than the wavelength) and by a multiple scattering medium (forest of steel rods) and shown in Fig. 2. After emitting a short pulse from the source, the sensors at the TRM collect the impulse response. The spread of these impulse responses is two orders of magnitude higer than the initial pulse duration as the multi-scattering medium is highly diffusive. As explained before, in the next step the responses are flipped in memory and re-transmitted from the TRM. The impulse duration, reconstructed at the original source is the same as the original; the spatial spread is a level of magnitude smaller (i.e. more coherent) when the propagation happens in the complex medium, than in the case of free space propagation.

2) The instantaneous time mirror approach 'à la Loschmidt': Going back to Loschmidt's demon able to turn the direction of particles instanteneously, if one decides to imitate it, unlike the Huygens case, the measurements of the incoming wavefield have to be performed at one specific time in the whole volume. At that point a new set of initial conditions is imposed, in which the sign of the time derivative is reversed, and the resulting wave is the time-reversed original.

Examine a case of a bath of fluid, placed on a shaker to control its vertical motion. After emitting a pulse from a 


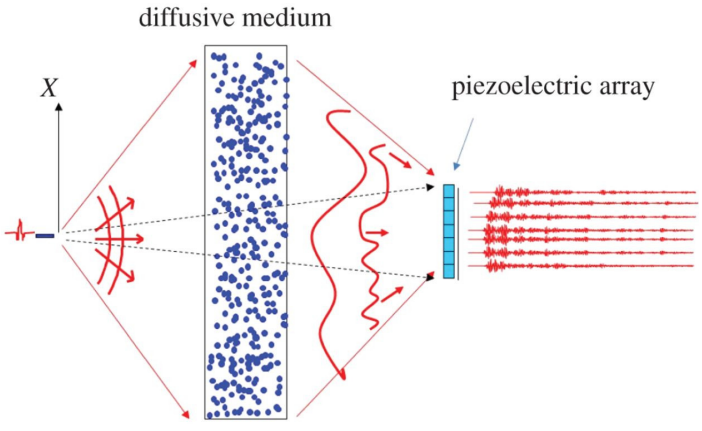

Fig. 2. Time-reversal experiment through a diffusive medium [3]

point on the fluid surface, at the chosen time instant a vertical downwards acceleration is applied to the bath, an impulsive change of wave celerity which can be described by a delta function in time. While the propagation of the initial outwards propagating wave is not affected, a new contribution emerges: a backwards converging circular wave packet. Just like in the Huygens case, this wave packet focuses at the original source and proceeds diverging afterwards. While the result is the same, we note that in this case no transceivers or memory elements were used: the information is stored in the medium itself.

\section{The Bridge to Computing}

Among time reversal studies that have followed the development of these concepts one creates a link between computation and time reversal in waves [7]. A dissipative chaotic system consisting of a drop bouncing on a vibrated liquid bath, exchanging information with the waves it forms, can be reversed. The elementary motions performed by the system are equivalent to writing, storing, reading and erasing operations of a Turing machine. The bouncing drop reads information as it backtracks, at the same time it is erasing the read information. In the next section, we investigate computational systems which use an equivalent principle to perform useful calculations and save power.

\section{B. Reversible Hardware}

Landauer famously concluded that "the information is physical" and brought together Shannon's and Boltzmann's views on entropy. [1] Digital computation that does not lose information (erase bits of information), does not have to dissipate power. When we delete a bit, the information that was stored there physically moves to the environment in form of heat, a direct display of Boltzmann's thermodinamical entropy. None of it would have to be dissipated from the entropical perspective if the erasure was not performed.

Observe a digital circuit consisting of logical gates: e.g. a single AND gate with its two inputs and one output. Its output is one when both inputs are one simultaneously; otherwise it is zero. Hence the knowledge about the output is not enough to tell us the inputs, as three different input combinations collapse into one output state. If we want to make an informationpreserving gate, it has to have a one-to-one correspondence between output states and input states. This asks for the same number of outputs and inputs in such reversible gates.

Two reversible bit operations are bit inversion and swap of two variables. To make more use of them, we devise gates controlling these operations according to the state of other variables. Fig. 3 shows some basic reversible gates as part of larger reversible circuits: all circuits in the figure are built using Feynman and Fredkin gates. Feynman gate is a controlled NOT: the variable with the $\oplus$ is inverted if and only if the control input, the variable with $\bullet$ is equal to 1 . In a more general setting of the Toffoli gate, multiple variables can control a single NOT; in that case the function controlling the gate is an AND of the control inputs. Similarly, the Fredkin gate swaps the variables joining in the $x$ if and only if the variable(s) with $\bullet$ are equal to 1 .

These gates enable design of reversible circuits which perform the usual digital electronics tasks. A full adder [8] and D-latch [9] are shown in Fig. 3. Additional inputs/outputs are auxilliary variables-the ancilla bits. In the D-latch example, the 0 bit and the Feynman gate attached to it are necessary to copy the latch output for feedback, as reversible circuits do not allow fan-out (it violates the one-to-one correspondence requirement).

Most classical computation is irreversible, re-using the memory by often removing intermediate results. In the past, most of the dissipation in logic circuits came from imperfections of the practical implementation. With the progress of semiconductor technology, the dissipation levels are approaching those of Landauer's limit, and reversibility is gaining importance. As Moore's law comes to its potential end, and alternative solutions are sought, one of the candidates being reversible computing. Irreversible calculations could be easily embedded in a reversible computation by merely keeping track of the steps made. To mitigate the need for unbounded memory, Bennett [2] introduced a trick: if a computation is made in a reversible circuit in one direction and then in reverse (computed, and then uncomputed), the memory occupied in the direct pass is freed in the return pass, and it is available for a new computation without bit erasures, and entropy is not increased (the memory after the return pass is back to the state before the direct pass).

The lack of application for reversible computation in the classical realm is reversed in the quantum computing domain. Most quantum computing schemes require reversibility to operate, so the reversible logic gates are constituent parts of quantum circuits (and are often referred to as quantum gates). Reversible computation is not limited to electronics (where adiabatic circuits are known for several decades [10]) and quantum computers: (micro)electromechanical systems and quantum dots are also capable of reversible computation.

\section{The DESIGN}

We have seen so far a computational paradigm relying on reversibility of calculation, and a communication scheme relying on the reversibility of wave propagation. In this part of the article, we proceed with designing a TRM based on reversible hardware. The reversible gates will form the digital 


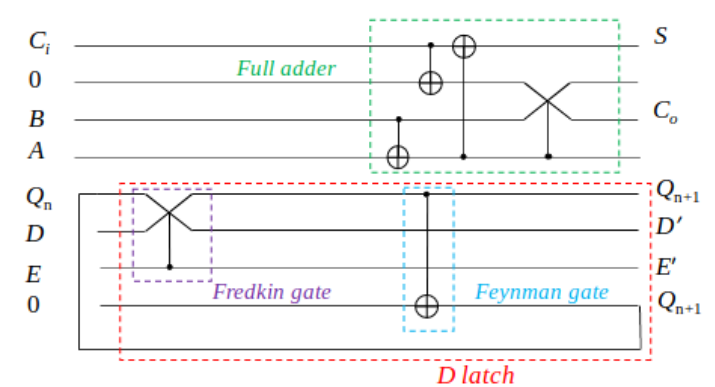

Fig. 3. Reversible circuits: D-latch, full adder, and logical gates as building blocks

logic part in the TRM, but the design is more complex than just digital logic. With Fig. 4 we illustrate the layers of the design task:

1) The environment is reversible to an extent. In the use case of acoustic underwater communication, the physics of wave propagation in water is reversible, but the issues arise as we lose information in the process: parts of the wave might end up reflected to unreachable parts of the environment if the observed space is not ergodic, guaranteeing all parts of the environment to be visited by the wave components. The hardware in contact with the environment are the microphones and speakers, i.e. sensors and actuators.

2) The analog computation part of the TRM loses information. It comprises of anti-alias filters before analog-todigital conversion (ADC), filters after digital-to-analog conversion (DAC), amplifiers accompanying the filters and the converters themselves, at the transition to the digital domain. We analyse these components in Section III-A.

3) Finally, the digital computation part of the TRM is reversible and no increase in entropy is necessary. This part entails writing in memory and unwriting, in the fashion of Bennett's trick, enabling reuse of memory for the next incoming wave, while not increasing the entropy. It may include a transform into frequency domain and digital filtering, and we discuss these options as well in Section III-B.

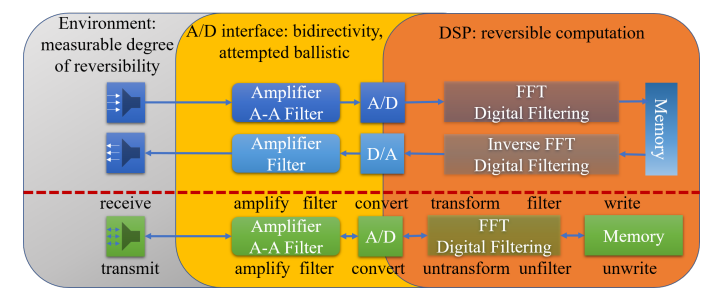

Fig. 4. The classical (top) and the reversible solution (bottom) for the classical time reversal chain.

\section{A. Analog processing}

Analog processing is the lossy, inherently irreversible bridge between two domains which exhibit reversibility: the physics of wave propagation and the digital signal processing chain we introduce. Our main goal at this point is to make the mechanisms in this part of the processing chain bidirectional, so that they can be used both for the inputs and the outputs. However, we are interested in saving as much information as we can, so we investigate the information loss in this part of the chain as well.

\section{1) Bidirectional amplification and $A D / D A$ conversion:} From the information-preserving perspective, ideal amplification is not an interesting process. However, the real amplifier is an imperfect device with a limited bandwidth and it loses signal information and introduces changes in the signal shape. It requires additional energy for the signal, hence we have to allocate a non-zero energy budget for this part of the computation. At the same time, the analog to digital and digital to analog converters both modify the signal they convert due to finite resolution and sampling rates, losing information about the original signal. However, the idea of the single device performing as both an ADC and a DAC depending on the direction exists both in academia and industry, with a large number of patents describing these bi-directional devices. [11] In our proposed solution, we assume that the bi-directional converters are bundled with bi-directional amplifiers [12]. We note the complexity of structure and switching in these devices.

2) The information-increasing filter: A part of analog to digital conversion is the anti-aliasing filter. Filtering, in the most common interpretation, removes a part of the signal and hence loses information about the original signal. However, the anti-aliasing filter is employed to prevent significant information loss due to spectrum overlaps in the analog-todigital conversion, and hence in this situation represents an information gain and may be re-interpreted in the context of useful, relevant information [13]. To avoid confusion, we may consider the anti-aliasing filter as a part of the analog-todigital converter and as such, implement it in the bi-directional fashion.

3) One-bit reversal: The conversion is additionally simplified in the one-bit solution [14] where the receivers at the mirror register only the sign of the waveform and the transmitters emit the reversed version based on this information. It is a special case of analog-to-digital and digital-to-analog conversion with single bit converters. The reduction in discretisation levels also means simplification of the processing chain and making its reversal (bi-directivity) even simpler. The question of the information loss is not straightforward: while the information about the incoming wave is lost in the conversion process (and the loss is maximal due to minimal resolution), spatial and temporal resolution are not significantly degraded.

This scheme can also be called "one-trit" reversal: there are three possible states in the practical implementation: positive pressure, negative pressure, and 'off'. Reversibility and multivalued logic were going hand in hand from the beginning: binary reversible logic is just a special case of multi-valued reversible logic. Hence, this scheme is readily implementable in reversible logic as well. 


\section{B. Digital processing}

With the functionalities reversible gates presented in Section II-B can offer when combined into logical circuits, building a digital signal processing chain for wave time reversal becomes the matter of combining circuits into more complex structures, akin to traditional circuit design. The idea we show here can easily be translated into any scheme of modulationdemodulation, coding-decoding, which are often seen in communications hardware and software. While fully functional and directly applicable, our time reversal signal processing chain is a proof of concept for reversible communications signal processing of arbitrary complexity.

1) Time domain reversal: The first, straightforward way of performing time reversal of a digitally sampled wave is storing it in memory and reading the samples in the reverse order (last in, first out, LIFO), analoguous to storing the samples on the stack. The design of registers in reversible logic is a wellexplored topic [9] and both serial and parallel reading/writing can be implemented. In the sense of already presented circuits, we have seen a design for the D-latch (Fig. 3): a combination of latches makes a flip-flop, and a series of flip flops makes a register (and a reversible address counter). In the case of wave time reversal, this is important to know, as the two possible variants of wave time reversal can be interpreted as two variants of memory writing:

1) Wave reversal à la Loschmidt is a large register being loaded in parallel with wave data;

2) Wave reversal à la Huygens is a large register being loaded serially with wave data.

In the case of a localized time reversal mirror (all samples at the same place) $m$ bits from the ADC are memorised at the converter's sample rate inside a $k \times m$ bit register matrix (where $k$ is the number of samples to be stored for time reversal). In the receiving process, the bits are stored, in the transmission process they are unstored, returning the memory into the blank state it started from (uncomputation). We utilise Bennett's trick and lose information without the entropic penalty: the information is kept as long as it is relevant.

2) Frequency domain reversal: When additional signal processing, e.g. filtering or modulation is performed, it is convenient to reverse waves in frequency domain: there, time domain reversal is achieved by phase conjugation, i.e. changing the sign of the signal's phase. The transition from time to frequency domain (and vice versa) in digital domain is performed by the Fast Fourier Transform (FFT) and its inverse counterpart. These procedures are inherently reversible and information-preserving, and their implementation in reversible digital circuits asks for a network of reversible adders (and reversible multipliers, again comprised of adders) [8], and we have already seen an implementation of a reversible adder in Fig. 3. The necessary phase conjugation is an arithmetic operation of sign reversal, again perfectly reversible and with a known implementation. The additional signal processing can be performed reversibly as well: one example is the filtering process done through filter banks and wavelet transforms. It remains reversible as all components of signals are preserved, if nothing then as the remainder [15]. Both the reversible wavelet computation and reversible Fast Fourier Transform use the lifting scheme.

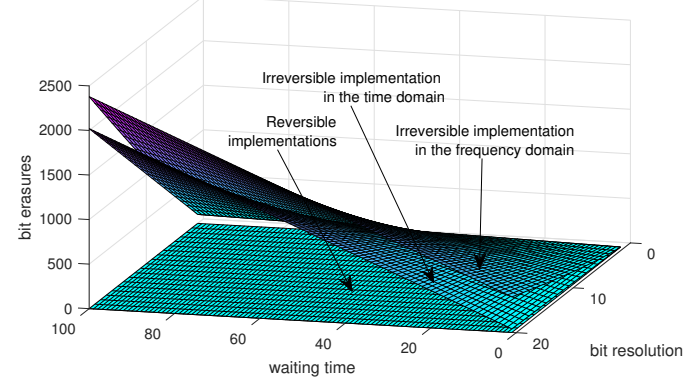

(a)

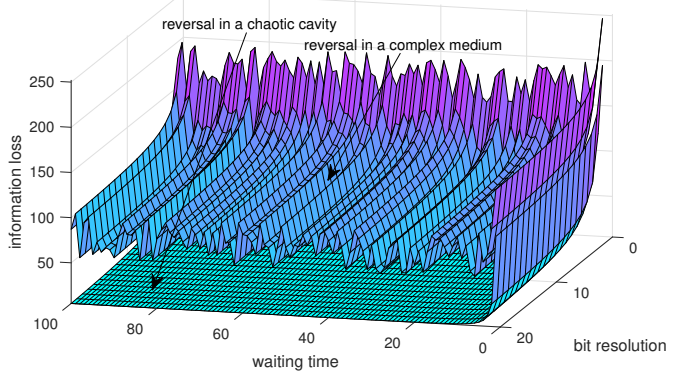

(b)

Fig. 5. Information loss in (a) digital and (b) analog part of the system. Units are omitted as the particular aspects of implementation are not relevant for the illustration of effects. Plot (a) is obtained by counting operations, plot (b) by simulation of back-scattering, both originating from theoretical calculations.

\section{Discussion AND CONClusions}

We have presented the components to be used in the implementation: where possible (in the digital domain) we use reversible circuits, otherwise we use bidirectional components. What is the gain of the new implementation? As already suggested, the loss of information is directly related to the dissipation of energy in the system, so let us observe how does our solution fare in this regard.

Fig. 5(a) is a comparison of the bit erasures in different implementations of the digital circuitry: frequency domain (FFT) and time domain reversal performed by irreversible circuits, compared to reversible implementations. The number of erasures changes depending on two parameters: bit resolution of the ADC and the waiting time-the length of the interval in which samples are collected before reversal starts, equivalent to the number of digitised samples. The increase in both means additional memory locations and additional dissipation for irreversible circuits. The irreversible FFT implementation has an additional information loss caused by additional irreversible circuitry compared to the irreversible time domain implementation. Our implementation has no bit erasures whatsoever. The price that is paid reflects in the larger number of gates used in the circuit: the number of gates has only spatial consequences, information-related energy dissipation is zero thanks to information conservation. 
Having no bit erasures means theoretical decrease in energy consumption proportional to the number of bits erased by the state of the art irreversible implementations-multiplied by the number of processing chains serving multiple transceivers, it is clear that this quantity, as small as it may seem in the case of a single transceiver, is indeed significant, especially in the near future where the Landauer limit becomes the dominant bound in semiconductor component power dissipation. As the circuits in our solution perform arithmetical and logical operations in the same vein as irreversible circuits in the state of the art implementations, the performance of the two solutions is the same in terms of results (the components are validated at the level of logical circuits).

On the other hand, in Fig. 5(b) we see the information loss in the analog part of the system, and we differentiate two typical environments, the chaotic cavity and the complex medium. The chaotic cavity is an ergodic space with sensitive dependence on initial conditions for waves. In such an environment there is little to no loss in the information if the waiting time is long enough and the ADC resolution is high enough. The complex medium is one with a large number of scatterers; In such media, the difference is caused by some of the wave components being reflected backwards by the scattering environment, hence not reaching the TRM. Again, more information is retained with the increase in the ADC resolution. However, as reported in [14], the information loss from low-resolution ADC use does not affect the performance of the algorithm. The analog part of the scheme remains a topic of our future work, as it leaves space for improvements of the scheme.

When the first prototype of a reversible FFT chip was introduced [8], it was shown that the implementation based on 8-bit adders and 11-bit multipliers requires 40,000 transistors. As the idea of information and energy conservation in computation allows for very dense packing (no heat dissipation to limit the density), this order of magnitude for our solution is acceptible (the rest of the circuitry we introduce in the chain needs two or three orders of magnitude fewer transistors), and if we opt for no frequency domain processing, i.e. just using memory, we can perform the task with less than 1,000 transistors.

In this first application of reversible hardware to a physical process we have demonstrated the complementary nature of wave reversal and computational reversal which can be put to use. Our implementation of wave time reversal using reversible hardware carries the promise of reduced power consumption, and it also fits in the bigger picture: in this vision of the future, all computation is reversible, no matter if it is performed on classical or quantum basis.

This solution is just the first step in the proliferation of reversible computation in communications. The inherent reversible properties of communications, including but not limited to channel reciprocity and transmitter-receiver duality, make communications technology an area with a lot of potential in reversible computation. With the inevitable penetration of quantum-based techniques in communications, this link with reversible computation grows stronger and needs to be thoroughly investigated.

\section{REFERENCES}

[1] R. Landauer, "Irreversibility and Heat Generation in the Computing Process," IBM Journal of Research and Development, vol. 5, no. 3, pp. 183-191, Jul. 1961

[2] C. H. Bennett, "Logical Reversibility of Computation," IBM Journal of Research and Development, vol. 17, no. 6, pp. 525-532, Nov. 1973.

[3] M. Fink, "From loschmidt daemons to time-reversed waves," Philosophical Transactions of the Royal Society A: Mathematical, Physical and Engineering Sciences, vol. 374, no. 2069, p. 20150156, 2016.

[4] — "Time reversal of ultrasonic fields. I. Basic principles," IEEE Transactions on Ultrasonics, Ferroelectrics, and Frequency Control, vol. 39, no. 5, pp. 555-566, Sep. 1992.

[5] J. de Rosny and M. Fink, "Overcoming the diffraction limit in wave physics using a time-reversal mirror and a novel acoustic sink," Physical review letters, vol. 89, no. 12, p. 124301, 2002.

[6] A. Derode, P. Roux, and M. Fink, "Robust acoustic time reversal with high-order multiple scattering," Physical review letters, vol. 75, no. 23, p. 4206, 1995 .

[7] S. Perrard, E. Fort, and Y. Couder, "Wave-Based Turing Machine: Time Reversal and Information Erasing," Physical Review Letters, vol. 117, no. 9, p. 094502, Aug. 2016. [Online]. Available: https://link.aps.org/doi/10.1103/PhysRevLett.117.094502

[8] M. Skoneczny, Y. V. Rentergem, and A. D. Vos, "Reversible fourier transform chip," in 2008 15th International Conference on Mixed Design of Integrated Circuits and Systems, Jun. 2008, pp. 281-286.

[9] N. M. Nayeem, M. A. Hossain, L. Jamal, and H. M. H. Babu, "Efficient Design of Shift Registers Using Reversible Logic,' in 2009 International Conference on Signal Processing Systems, May 2009, pp. 474-478.

[10] M. P. Frank, "Asynchronous ballistic reversible computing," in 2017 IEEE International Conference on Rebooting Computing (ICRC). IEEE, 2017, pp. 1-8.

[11] R. E. Suarez-Gartner, "Reversible analog/digital (digital/analog) converter,' Sep. 16 1975, uS Patent 3,906,488.

[12] M. Azadmehr and Y. Berg, "A bi-directional autozeroing amplifier for designing bi-directional time-continuous frequency mixer/extractor," in TENCON 2009-2009 IEEE Region 10 Conference. IEEE, 2009, pp. $1-4$.

[13] B. C. Geiger and G. Kubin, Information Loss in Deterministic Signal Processing Systems, ser. Understanding Complex Systems. Springer International Publishing, 2018. [Online]. Available: //www.springer. $\mathrm{com} / \mathrm{gp} / \mathrm{book} / 9783319595320$

[14] A. Derode, A. Tourin, and M. Fink, "Ultrasonic pulse compression with one-bit time reversal through multiple scattering," Journal of Applied Physics, vol. 85, no. 9, pp. 6343-6352, Apr. 1999. [Online]. Available: https://aip.scitation.org/doi/abs/10.1063/1.370136

[15] T. Suzuki, N. Tanaka, and H. Kudo, "Redefined block-lifting-based filter banks with efficient reversible nonexpansive convolution," IEEE Transactions on Circuits and Systems for Video Technology, vol. 29, no. 5, pp. 1438-1447, 2018.

Harun Siljak (M '15) graduated from Automatic Control and Electronics Department, University of Sarajevo (BoE 2010, MoE 2012) and International Burch University Sarajevo ( $\mathrm{PhD} 2015)$. Currently he is a postdoctoral Marie Curie Fellow at CONNECT Centre, Trinity College Dublin, working on reversible computation, complex and nonlinear dynamics in wireless communications.

Julien de Rosny received the M.S. Degree and the Ph.D. degree from the University UPMC, Paris, France in 1996 and 2000, respectively, in wave physics. He was a postdoctoral researcher at Scripps Research Institute, California, USA, in 2000-2001. In 2001, he joined CNRS at Laboratoire Ondes et Acoustique, France. Since 2014, he is a CNRS senior scientist at Institut Langevin, Paris, France. His research interests include telecommunications in complex media, acoustic and electromagnetic waves based imaging.

Mathias Fink is the George Charpak Professor at ESPCI Paris where he founded in 1990 the Laboratory "Ondes et Acoustique" that became in 2009 the Langevin Institute. He is member of the French Academy of Science and of the National Academy of Technologies of France. In 2008, he was elected at the College de France on the Chair of Technological Innovation. His area of research is concerned with the propagation of waves in complex media and the development of numerous instruments based on this basic research. His current research interests include time-reversal in physics, wave control in complex media, super-resolution, metamaterials, multiwave imaging, geophysics and telecommunications. He holds more than 70 patents, and has published more than 400 peer reviewed papers and book chapters. 6 start-up companies with more than 400 employees have been created from his research (Echosens, Sensitive Object, Supersonic Imagine, Time Reversal Communications, CardiaWave and GreenerWave). 